PROCEEDINGS OF THE

AMERICAN MATHEMATICAL SOCIETY

Volume 130, Number 12, Pages 3483-3493

S 0002-9939(02)06597-8

Article electronically published on May 9, 2002

\title{
ON SYZYGIES OF SEGRE EMBEDDINGS
}

\author{
ELENA RUBEI
}

(Communicated by Michael Stillman)

\begin{abstract}
We study the syzygies of the ideals of the Segre embeddings. Let $d \in \mathbf{N}, d \geq 3$; we prove that the line bundle $\mathcal{O}(1, \ldots, 1)$ on the $P^{1} \times \ldots \times P^{1}$ ( $d$ copies) satisfies Property $N_{p}$ of Green-Lazarsfeld if and only if $p \leq 3$. Besides we prove that if we have a projective variety not satisfying Property $N_{p}$ for some $p$, then the product of it with any other projective variety does not satisfy Property $N_{p}$. From this we also deduce other corollaries about syzygies of Segre embeddings.
\end{abstract}

\section{INTRODUCTION}

Let $L$ be a very ample line bundle on a smooth complex projective variety $Y$ and let $\varphi_{L}: Y \rightarrow \mathbf{P}\left(H^{0}(Y, L)^{*}\right)$ be the map associated to $L$. We recall the definition of Property $N_{p}$ of Green-Lazarsfeld, studied for the first time by Green in [Gr1-2] (see also G-L], Gr3]):

Let $Y$ be a smooth complex projective variety and let $L$ be a very ample line bundle on $Y$ defining an embedding $\varphi_{L}: Y \hookrightarrow \mathbf{P}=\mathbf{P}\left(H^{0}(Y, L)^{*}\right)$; set $S=S(L)=$ Sym $^{*} H^{0}(L)$, the homogeneous coordinate ring of the projective space $\mathbf{P}$, and consider the graded $S$-module $G=G(L)=\bigoplus_{n} H^{0}\left(Y, L^{n}\right)$; let $E_{*}$

$$
0 \longrightarrow E_{n} \longrightarrow E_{n-1} \longrightarrow \ldots \longrightarrow E_{0} \longrightarrow G \longrightarrow 0
$$

be a minimal graded free resolution of $G$; the line bundle $L$ satisfies Property $N_{p}$ $(p \in \mathbf{N})$ if and only if

$$
\begin{aligned}
& E_{0}=S, \\
& E_{i}=\bigoplus S(-i-1) \quad \text { for } 1 \leq i \leq p .
\end{aligned}
$$

(Thus $L$ satisfies Property $N_{0}$ if and only if $Y \subset \mathbf{P}\left(H^{0}(L)^{*}\right)$ is projectively normal, i.e. $L$ is normally generated; $L$ satisfies Property $N_{1}$ if and only if $L$ satisfies Property $N_{0}$ and the homogeneous ideal $I$ of $Y \subset \mathbf{P}\left(H^{0}(L)^{*}\right)$ is generated by quadrics; $L$ satisfies Property $N_{2}$ if and only if $L$ satisfies Property $N_{1}$ and the module of syzygies among quadratic generators $Q_{i} \in I$ is spanned by relations of the form $\sum L_{i} Q_{i}=0$, where $L_{i}$ are linear polynomials; and so on.)

Now let $L=\mathcal{O}_{\mathbf{P}^{n_{1}} \times \ldots \times \mathbf{P}^{n_{d}}}\left(a_{1}, \ldots, a_{d}\right)$, where $d, a_{1}, \ldots, a_{d}, n_{1}, \ldots, n_{d}$ are positive integers. Among the papers on syzygies in this case we quote $[\mathrm{B}-\mathrm{M}], \mathrm{Gr} 1-2, \mathrm{O}-\mathrm{P}$, J-P-W], G-P], [Las, and $\mathrm{P}-\mathrm{W}]$. In the first one, the authors examined the cases in which the resolution is "pure", i.e. the minimal generators of each module of

Received by the editors December 20, 2000 and, in revised form, July 13, 2001.

2000 Mathematics Subject Classification. Primary 14M25, 13 D02.

(C)2002 American Mathematical Society 
syzygies have the same degree. We quote the following results from the other papers:

Case $d=1$, i.e. the case of the Veronese embedding:

Theorem 1 (Green Gr1-2]). Let a be a positive integer. The line bundle $\mathcal{O}_{\mathbf{P}^{n}}(a)$ satisfies Property $N_{a}$.

Theorem 2 (Ottaviani-Paoletti $[\mathrm{O}-\mathrm{P}]$ ). If $n \geq 2, a \geq 3$ and the bundle $\mathcal{O}_{\mathbf{P}^{n}}(a)$ satisfies Property $N_{p}$, then $p \leq 3 a-3$.

Theorem 3 (Josefiak-Pragacz-Weyman [J-P-W]). The bundle $\mathcal{O}_{\mathbf{P}^{n}}(2)$ satisfies Property $N_{p}$ if and only if $p \leq 5$ when $n \geq 3$ and for all $p$ when $n=2$.

(See $\mathrm{O}-\mathrm{P}$ for a more complete bibliography.)

Case $d=2$ :

Theorem 4 (Gallego-Purnapranja [G-P]). Let $a, b \geq 2$. The line bundle $\mathcal{O}_{\mathbf{P}^{1} \times \mathbf{P}^{1}}(a, b)$ satisfies Property $N_{p}$ if and only if $p \leq 2 a+2 b-3$.

Theorem 5 (Lascoux-Pragacz-Weymann $\left[\mathrm{Las},[\mathrm{P}-\mathrm{W}]\right.$ ). Let $n_{1}, n_{2} \geq 2$. The line bundle $\mathcal{O}_{\mathbf{P}^{n_{1}} \times \mathbf{P}^{n_{2}}}(1,1)$ satisfies Property $N_{p}$ if and only if $p \leq 3$.

Here we consider $\mathcal{O}(1, \ldots, 1)$ on $\mathbf{P}^{1} \times \ldots \times \mathbf{P}^{1}(d$ times, for any $d)$. We prove (Section 2):

Theorem 6. The line bundle $\mathcal{O}(1, \ldots, 1)$ on $\mathbf{P}^{1} \times \ldots \times \mathbf{P}^{1}$ (d times) satisfies Property $N_{3}$ for any $d$.

Besides we prove (Section 3):

Proposition 7. Let $X$ and $Y$ be two projective varieties and let $L$ be a line bundle on $X$ and $M$ a line bundle on $Y$. Let $\pi_{X}: X \times Y \rightarrow X$ and $\pi_{Y}: X \times Y \rightarrow Y$ be the canonical projections. Suppose $L$ and $M$ satisfy Property $N_{1}$. Let $p \geq 2$. If $L$ does not satisfy Property $N_{p}$, then $\pi_{X}^{*} L \otimes \pi_{Y}^{*} M$ does not satisfy Property $N_{p}$, either.

Corollary 8. Let $a_{1}, \ldots, a_{d}$ be positive integers with $a_{1} \leq a_{2} \leq \ldots \leq a_{d}$. Suppose $k=\max \left\{i \mid a_{i}=1\right\}$. If $k \geq 3$ the line bundle $\mathcal{O}_{\mathbf{P}^{n_{1}} \times \ldots \times \mathbf{P}^{n_{d}}}\left(a_{1}, \ldots, a_{d}\right)$ does not satisfy Property $N_{4}$ and if $d-k \geq 2$ it does not satisfy Property $N_{2 a_{k+1}+2 a_{k+2}-2}$.

In particular, from Corollary 8 and Theorem [6, we have:

Corollary 9. Let $d \geq 3$. The line bundle $\mathcal{O}_{\mathbf{P}^{1} \times \ldots \times \mathbf{P}^{1}}(1, \ldots, 1)$ (d times) satisfies Property $N_{p}$ if and only if $p \leq 3$.

\section{Proof of Theorem 6}

First we have to recall some facts on toric ideals from [St].

Let $k \in \mathbf{N}$. Let $A=\left\{a_{1}, \ldots, a_{n}\right\}$ be a subset of $\mathbf{Z}^{k}$. The toric ideal $\mathcal{I}_{A}$ is defined as the ideal in $\mathbf{C}\left[x_{1}, \ldots, x_{n}\right]$ generated as vector space by the binomials

$$
x_{1}^{u_{1}} \ldots x_{n}^{u_{n}}-x_{1}^{v_{1}} \ldots x_{n}^{v_{n}}
$$

for $\left(u_{1}, \ldots, u_{n}\right),\left(v_{1}, . ., v_{n}\right) \in \mathbf{N}^{n}$, with $\sum_{i=1, \ldots, n} u_{i} a_{i}=\sum_{i=1, \ldots, n} v_{i} a_{i}$.

We have that $\mathcal{I}_{A}$ is homogeneous if and only if $\exists \omega \in \mathbf{Q}^{k}$ s.t. $\omega \cdot a_{i}=1$ $\forall i=1, \ldots, n$; the rings $\mathbf{C}\left[x_{1}, \ldots, x_{n}\right]$ and $\mathbf{C}\left[x_{1}, \ldots, x_{n}\right] / \mathcal{I}_{A}$ are multigraded by $\mathbf{N} A$ via $\operatorname{deg} x_{i}=a_{i}$; the element $x_{1}^{u_{1}} \ldots x_{n}^{u_{n}}$ has multidegree $b=\sum_{i} u_{i} a_{i} \in \mathbf{N} A$ and degree $\sum_{i} u_{i}=b \cdot \omega$; we define $\operatorname{deg} b=b \cdot \omega$. 
For each $b \in \mathbf{N} A$, let $\Delta_{b}$ be the simplicial complex on the set $\{1, \ldots, n\}$ defined as follows:

$$
\Delta_{b}=\left\{F \subset\{1, \ldots, n\}: b-\sum_{i \in F} a_{i} \in \mathbf{N} A\right\}
$$

(thus, by identifying $\{1, \ldots, n\}$ with $A$, we have:

$$
\Delta_{b}=\bigcup_{k \in \mathbf{N}, a_{i_{1}}, \ldots, a_{i_{k}} \in A, a_{i_{1}}+\ldots+a_{i_{k}}=b}\left\langle a_{i_{1}}, \ldots, a_{i_{k}}\right\rangle,
$$

where $\left\langle a_{i_{1}}, \ldots, a_{i_{k}}\right\rangle$ is the simplex generated by $\left.a_{i_{1}}, \ldots, a_{i_{k}}\right)$.

The following theorem studies the syzygies of the ideal $\mathcal{I}_{A}$; it was proved by Campillo and Marijuan for $k=1$ in [C-M] and by Campillo and Pison for general $k$ and $j=0$ in $[\mathrm{C}-\mathrm{P}$; the following more general statement is due to Sturmfels (Theorem 12.12 p. 120 in $[\underline{\mathrm{St}}]$ ).

Theorem 10 (see $\mathrm{St}$ and also $[\mathrm{C}-\mathrm{M}], \mathrm{C}-\mathrm{P}$ ). Let $A=\left\{a_{1}, \ldots, a_{n}\right\}$ be a subset of $\mathbf{N}^{k}$ and $\mathcal{I}_{A}$ be the associated toric ideal. Let $0 \rightarrow E_{n} \rightarrow \ldots \rightarrow E_{1} \rightarrow E_{0} \rightarrow G \rightarrow 0$ be a minimal free resolution of $G=\mathbf{C}\left[x_{1}, \ldots, x_{n}\right] / \mathcal{I}_{A}$ on $\mathbf{C}\left[x_{1}, \ldots, x_{n}\right]$. Each of the generators of $E_{j}$ has a unique multidegree. The number of the generators of multidegree $b \in \mathbf{N} A$ of $E_{j+1}$ equals the rank of the $j$-th reduced homology group $\tilde{H}_{j}\left(\Delta_{b}, \mathbf{C}\right)$ of the simplicial complex $\Delta_{b}$.

Notation 11. If $\alpha$ is a chain in a topological space, $\operatorname{sp}(\alpha)$ will denote the support of $\alpha$, i.e. the union of the supports of the simplexes $\sigma_{i}$ s.t. $\alpha=\sum_{i} c_{i} \sigma_{i}, c_{i} \in \mathbf{Z}$. If $X$ is a simplicial complex, $s k^{i}(X)$ will denote the $i$-skeleton of $X$.

Proof of Theorem [6. If we take $A=A_{d}=\left\{\left(1, \epsilon_{1}, \ldots, \epsilon_{d}\right) \mid \epsilon_{i} \in\{0,1\}\right\}$, we have that $\mathcal{I}_{A_{d}}$ is the ideal of the Segre embedding of $\mathbf{P}^{1} \times \ldots \times \mathbf{P}^{1}$ (d times), i.e. the ideal of the embedding of $\mathbf{P}^{1} \times \ldots \times \mathbf{P}^{1}$ ( $d$ times) by the line bundle $\mathcal{O}(1, \ldots, 1)$. In fact the ideal of the Segre embedding is generated as vector space by the homogeneous equations of the form

$$
\prod_{\epsilon_{1}, \ldots, \epsilon_{d} \in\{0,1\}} x_{\epsilon_{1}, \ldots, \epsilon_{d}}^{v_{\epsilon_{1}, \ldots, \epsilon_{d}}}-\prod_{\epsilon_{1}, \ldots, \epsilon_{d} \in\{0,1\}} x_{\epsilon_{1}, \ldots, \epsilon_{d}}^{u_{\epsilon_{1}}, \ldots, \epsilon_{d}}=0
$$

with $u_{\epsilon_{1}, \ldots, \epsilon_{d}}, v_{\epsilon_{1}, \ldots, \epsilon_{d}} \in \mathbf{N}$ and

$$
\sum_{\epsilon_{1}, \ldots, \epsilon_{d} \in\{0,1\}} v_{\epsilon_{1}, \ldots, \epsilon_{d}}\left(\epsilon_{1}, \ldots, \epsilon_{d}\right)=\sum_{\epsilon_{1}, \ldots, \epsilon_{d} \in\{0,1\}} u_{\epsilon_{1}, \ldots, \epsilon_{d}}\left(\epsilon_{1}, \ldots, \epsilon_{d}\right) .
$$

The last condition is equivalent to

$$
\sum_{\epsilon_{1}, \ldots, \epsilon_{d} \in\{0,1\}} v_{\epsilon_{1}, \ldots, \epsilon_{d}}\left(1, \epsilon_{1}, \ldots, \epsilon_{d}\right)=\sum_{\epsilon_{1}, \ldots, \epsilon_{d} \in\{0,1\}} u_{\epsilon_{1}, \ldots, \epsilon_{d}}\left(1, \epsilon_{1}, \ldots, \epsilon_{d}\right)
$$

(the equality of the first coordinate gives the homogeneity).

In this case $\omega=\omega_{d}=(1,0, \ldots, 0)(0$ repeated $d$ times $)$ and $n=2^{d}$.

Let $b \in \mathbf{N} A_{d}$; we have that $\operatorname{deg} b=(=b \cdot \omega)=k$ if and only if $b$ is the sum of $k$ (not necessarily distinct) elements of $A_{d}$. By identifying the set $\left\{1, \ldots, 2^{d}\right\}$ with $A_{d}$, we have that, if $k=\operatorname{deg} b, \Delta_{b}=\bigcup_{a_{i_{1}}, \ldots, a_{i_{k}} \in A_{d}, a_{i_{1}}+\ldots+a_{i_{k}}=b}\left\langle a_{i_{1}}, \ldots, a_{i_{k}}\right\rangle$; we say that $\left\langle a_{i_{1}}, \ldots, a_{i_{k}}\right\rangle$ is a degenerate $(k-1)$-simplex if $\exists l, m \in\{1, \ldots, k\}$ with $l \neq m$ s.t. $a_{i_{l}}=a_{i_{m}}$; thus $\Delta_{b}$ is equal to the union of the (possibly degenerate) $(k-1)$-simplexes $S$ with vertices in $A_{d}$ such that the sum of the vertices (with multiplicities) of $S$ is $b$. 
By Theorem 10, in order to prove that $\mathcal{O}_{\mathbf{P}^{1} \times \ldots \times \mathbf{P}^{1}}(1, \ldots, 1)\left(d\right.$ times) satisfies $N_{2}$, we have to prove that $H_{1}\left(\Delta_{b}\right)=0$ for each $b \in \mathbf{N} A_{d}$ with $\operatorname{deg} b \geq 4$. Analogously in order to prove that $\mathcal{O}_{\mathbf{P}^{1} \times \ldots \times \mathbf{P}^{1}}(1, \ldots, 1)\left(d\right.$ times) satisfies $N_{3}$, we have to prove that $H_{2}\left(\Delta_{b}\right)=0$ for each $b \in \mathbf{N} A_{d}$ with $\operatorname{deg} b \geq 5$.

The proof is by induction on $d$. Observe that any $b^{\prime} \in \mathbf{N} A_{d+1}$ with $\operatorname{deg} b^{\prime}=k$ is equal to $\left(\begin{array}{l}b \\ \epsilon\end{array}\right)$ for some $b \in \mathbf{N} A_{d}$ with $\operatorname{deg} b=k$ and for some $\varepsilon \in\{0,1, \ldots, k\}$. Then, in order to prove $N_{2}$ we suppose (by induction) that $H_{1}\left(\Delta_{b}\right)=0 \forall b \in \mathbf{N} A_{d}$ with $\operatorname{deg} b=k, k \geq 4$, and we show that $H_{1}\left(\Delta_{\left(\begin{array}{l}b \\ \varepsilon\end{array}\right)}\right)=0$ for $\varepsilon \in\{0, \ldots, k\}$ and in order to prove $N_{3}$ we suppose (by induction) that $H_{2}\left(\Delta_{b}\right)=0 \forall b \in \mathbf{N} A_{d}$ with $\operatorname{deg} b=k$, $k \geq 5$, and we show that $H_{2}\left(\Delta_{\left(\begin{array}{l}b \\ \varepsilon\end{array}\right)}\right)=0$ for $\varepsilon \in\{0, \ldots, k\}$.

Observe that, if $\varepsilon \in\{0, k\}(k:=\operatorname{deg} b)$, then obviously $\Delta_{\left(\begin{array}{l}b \\ \varepsilon\end{array}\right)}$ and $\Delta_{b}$ are isomorphic; besides $\Delta_{\left(\begin{array}{c}b \\ k-\varepsilon\end{array}\right)}$ is isomorphic to $\Delta_{\left(\begin{array}{c}b \\ \varepsilon\end{array}\right)} \forall \varepsilon \in\{0, \ldots, k\}$ (the isomorphism is given by substituting 0 with 1 and 1 with 0 in the last coordinate). Thus we may consider only the cases $\varepsilon \in\left\{1, \ldots,\left[\frac{k}{2}\right]\right\}$.

First we need some preliminary notation and lemmas.

Notation 12. Let $S=\left\langle a_{1}, \ldots, a_{k}\right\rangle$ be a (possibly degenerate) $k$-1-simplex, $a_{i} \in A_{d}$. Let $\varepsilon \in\{0, \ldots, k\}$. We denote

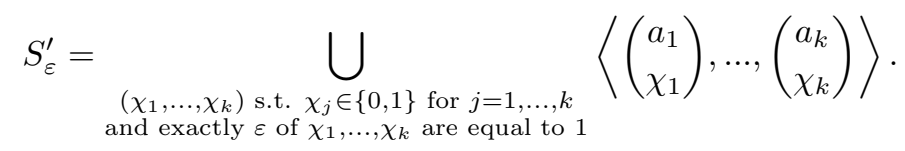

Example 13. Let $S=\left\langle a_{1}, a_{2}, a_{3}, a_{4}\right\rangle$ be a (possibly degenerate) tetrahedron, $a_{i} \in$ $A_{d}$. The set $S_{1}^{\prime}$ is the union of the four (possibly degenerate) tetrahedrons

$$
\begin{array}{ll}
\left\langle\left(\begin{array}{c}
a_{1} \\
1
\end{array}\right),\left(\begin{array}{c}
a_{2} \\
0
\end{array}\right),\left(\begin{array}{c}
a_{3} \\
0
\end{array}\right),\left(\begin{array}{c}
a_{4} \\
0
\end{array}\right)\right\rangle, & \left\langle\left(\begin{array}{c}
a_{1} \\
0
\end{array}\right),\left(\begin{array}{c}
a_{2} \\
1
\end{array}\right),\left(\begin{array}{c}
a_{3} \\
0
\end{array}\right),\left(\begin{array}{c}
a_{4} \\
0
\end{array}\right)\right\rangle, \\
\left\langle\left(\begin{array}{c}
a_{1} \\
0
\end{array}\right),\left(\begin{array}{c}
a_{2} \\
0
\end{array}\right),\left(\begin{array}{c}
a_{3} \\
1
\end{array}\right),\left(\begin{array}{c}
a_{4} \\
0
\end{array}\right)\right\rangle, & \left\langle\left(\begin{array}{c}
a_{1} \\
0
\end{array}\right),\left(\begin{array}{c}
a_{2} \\
0
\end{array}\right),\left(\begin{array}{c}
a_{3} \\
0
\end{array}\right),\left(\begin{array}{c}
a_{4} \\
1
\end{array}\right)\right\rangle .
\end{array}
$$

Thus $S_{1}^{\prime}$ can be obtained from $S$ by "constructing a tetrahedron on every one of the four faces of $S$ " and considering the union of these four tetrahedrons. The set $S_{2}^{\prime}$ is the union of the following six (possibly degenerate) tetrahedrons:

$$
\begin{array}{ll}
\left\langle\left(\begin{array}{c}
a_{1} \\
0
\end{array}\right),\left(\begin{array}{c}
a_{2} \\
0
\end{array}\right),\left(\begin{array}{c}
a_{3} \\
1
\end{array}\right),\left(\begin{array}{c}
a_{4} \\
1
\end{array}\right)\right\rangle, & \left\langle\left(\begin{array}{c}
a_{1} \\
0
\end{array}\right),\left(\begin{array}{c}
a_{2} \\
1
\end{array}\right),\left(\begin{array}{c}
a_{3} \\
0
\end{array}\right),\left(\begin{array}{c}
a_{4} \\
1
\end{array}\right)\right\rangle, \\
\left\langle\left(\begin{array}{c}
a_{1} \\
0
\end{array}\right),\left(\begin{array}{c}
a_{2} \\
1
\end{array}\right),\left(\begin{array}{c}
a_{3} \\
1
\end{array}\right),\left(\begin{array}{c}
a_{4} \\
0
\end{array}\right)\right\rangle, & \left\langle\left(\begin{array}{c}
a_{1} \\
1
\end{array}\right),\left(\begin{array}{c}
a_{2} \\
0
\end{array}\right),\left(\begin{array}{c}
a_{3} \\
0
\end{array}\right),\left(\begin{array}{c}
a_{4} \\
1
\end{array}\right)\right\rangle, \\
\left\langle\left(\begin{array}{c}
a_{1} \\
1
\end{array}\right),\left(\begin{array}{c}
a_{2} \\
0
\end{array}\right),\left(\begin{array}{c}
a_{3} \\
1
\end{array}\right),\left(\begin{array}{c}
a_{4} \\
0
\end{array}\right)\right\rangle, & \left\langle\left(\begin{array}{c}
a_{1} \\
1
\end{array}\right),\left(\begin{array}{c}
a_{2} \\
1
\end{array}\right),\left(\begin{array}{c}
a_{3} \\
0
\end{array}\right),\left(\begin{array}{c}
a_{4} \\
0
\end{array}\right)\right\rangle .
\end{array}
$$

Then $S_{2}^{\prime}$ can be obtained from $S$ by "constructing a tetrahedron on every one of the six edges of $S$ " and considering the union of these six tetrahedrons (see Figure 1 , representing $S_{\varepsilon}^{\prime}$ in the case $S$ is not degenerate).

Let $b \in \mathbf{N} A_{d}$ with $\operatorname{deg} b=k$ and $\varepsilon \in\{0, \ldots, k\}$. Obviously

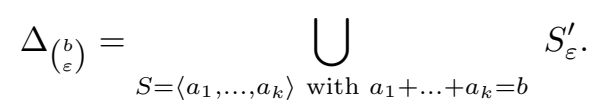



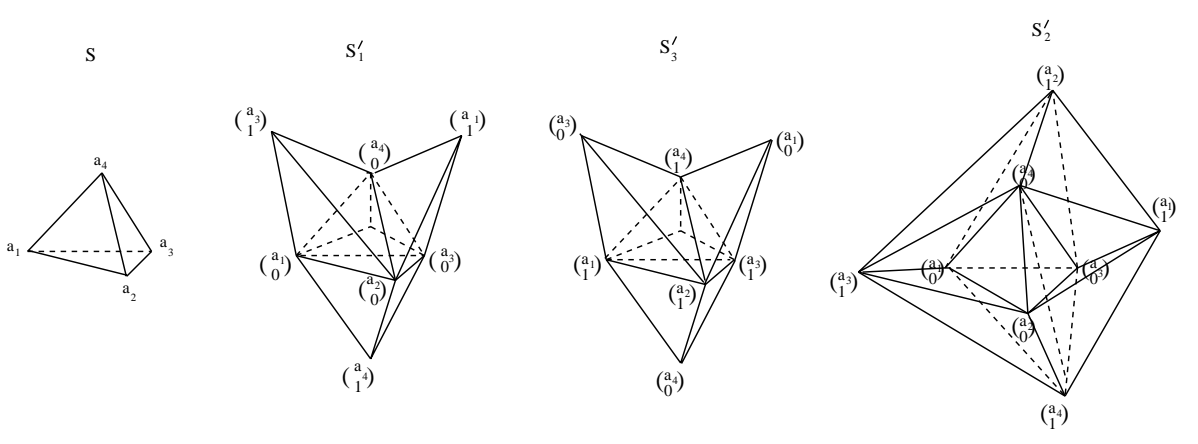

FiguRE 1.

Note to Figure 1. In the representation of $S_{2}^{\prime}$, for the sake of simplicity, we do not represent the tetrahedrons $\left\langle\left(\begin{array}{c}a_{1} \\ 0\end{array}\right),\left(\begin{array}{c}a_{2} \\ 1\end{array}\right),\left(\begin{array}{c}a_{3} \\ 0\end{array}\right),\left(\begin{array}{c}a_{4} \\ 1\end{array}\right)\right\rangle$ and $\left\langle\left(\begin{array}{c}a_{1} \\ 1\end{array}\right),\left(\begin{array}{c}a_{2} \\ 0\end{array}\right),\left(\begin{array}{c}a_{3} \\ 1\end{array}\right),\left(\begin{array}{c}a_{4} \\ 0\end{array}\right)\right\rangle$.

Notation 14. Let $b \in \mathbf{N} A_{d}$ with $\operatorname{deg} b=k$. For $l \in \mathbf{N}, 0 \leq l \leq k-1$, let

$$
F^{l}\left(\Delta_{b}\right)=\bigcup_{a_{1}, \ldots, a_{k} \in A_{d}} \text { s.t. } a_{1}+\ldots+a_{k}=b \quad \bigcup_{i_{0}, \ldots, i_{l} \in\{1, \ldots, k\}}\left\langle\left(\begin{array}{c}
a_{i_{0}} \\
0
\end{array}\right), \ldots,\left(\begin{array}{c}
a_{i_{l}} \\
0
\end{array}\right)\right\rangle .
$$

Observe that $F^{l}\left(\Delta_{b}\right) \subseteq \Delta_{\left(\begin{array}{l}b \\ \varepsilon\end{array}\right)}$ iff $k-\varepsilon \geq l+1$.

The idea of the proof is to consider an $l$-cycle (for $l=1,2)$ in $\Delta_{\left(\begin{array}{l}b \\ \varepsilon\end{array}\right)}$ and to show that it is homologous to an $l$-cycle in $F^{l}\left(\Delta_{b}\right)$ and then to show that it is homologous to 0 by using that $H_{l}\left(\Delta_{b}\right)=0$.

Remark 15. Let $S=\left\langle a_{1}, \ldots, a_{k}\right\rangle, a_{i} \in A_{d}$. If $k \geq 4$ and $1 \leq \varepsilon \leq k-2$, the set $S_{\varepsilon}^{\prime}$ contains the cone with vertex $\left(\begin{array}{c}a_{l} \\ 1\end{array}\right)$ on the border of $\left\langle\left(\begin{array}{c}a_{i_{1}} \\ 0\end{array}\right),\left(\begin{array}{c}a_{i_{2}} \\ 0\end{array}\right),\left(\begin{array}{c}a_{i_{3}} \\ 0\end{array}\right)\right\rangle$ for any $i_{1}, i_{2}, i_{3}, l \in\{1, \ldots, k\}$, with $l \neq i_{j}$ for $j=1,2,3$. This is true in particular if $k \geq 4$ and $\varepsilon \in\left\{1, \ldots,\left[\frac{k}{2}\right]\right\}$.

If $k \geq 5$ and $1 \leq \varepsilon \leq k-3$, the set $S_{\varepsilon}^{\prime}$ contains the cone with vertex $\left(\begin{array}{c}a_{l} \\ 1\end{array}\right)$ on the border of $\left\langle\left(\begin{array}{c}a_{i_{1}} \\ 0\end{array}\right), \ldots,\left(\begin{array}{c}a_{i_{4}} \\ 0\end{array}\right)\right\rangle$ for any $i_{1}, \ldots, i_{4}, l \in\{1, \ldots, k\}$ with $l \neq i_{j}$ for $j=1,2,3,4$. This is true in particular if $k \geq 5$ and $\varepsilon \in\left\{1, \ldots,\left[\frac{k}{2}\right]\right\}$.

Definition 16. For any $c \in \mathbf{N} A_{d}$ with $\operatorname{deg} c=s$ and $\varepsilon \in\{1, \ldots, s\}$, we define $R_{c, \varepsilon}$ to be the following set:

$$
\bigcup_{\substack{\alpha_{1}, \ldots, \alpha_{s} \in A_{d} \\
\text { s.t. } \alpha_{1}+\ldots+\alpha_{s}=c}} \bigcup_{\substack{i_{1}, \ldots, i_{s-1} \in\{1, \ldots, s\} \\
i_{l} \neq i_{m}}}\left\langle\left(\begin{array}{c}
\alpha_{i_{1}} \\
1
\end{array}\right), \ldots,\left(\begin{array}{c}
\alpha_{i_{\varepsilon}-1} \\
1
\end{array}\right),\left(\begin{array}{c}
\alpha_{i_{\varepsilon}} \\
0
\end{array}\right), \ldots,\left(\begin{array}{c}
\alpha_{i_{s-1}} \\
0
\end{array}\right)\right\rangle .
$$

Lemma 17. Let $c \in \mathbf{N} A_{d}$ with $\operatorname{deg} c=s$. We have that $\tilde{H}_{i}\left(\Delta_{\left(\begin{array}{c}c \\ \varepsilon-1\end{array}\right)}\right)=0 \mathrm{im}$ plies $\tilde{H}_{i}\left(R_{c, \varepsilon}\right)=0$ if we are in one of the following cases: a) $i=0, s \geq 3$, $\varepsilon \in\left\{1, \ldots,\left[\frac{s+1}{2}\right]\right\}$; b) $i=1, s \geq 4, \varepsilon \in\left\{1, \ldots,\left[\frac{s+1}{2}\right]\right\}$.

Proof. Observe that $R_{c, \varepsilon} \subseteq \Delta_{\left(\begin{array}{c}c \\ \varepsilon-1\end{array}\right)}$. Since $\tilde{H}_{i}\left(\Delta_{\left(\begin{array}{c}c \\ \varepsilon-1\end{array}\right)}\right)=0$, we have

$$
\tilde{H}_{i}\left(s k^{i+1}\left(\Delta_{\left(\begin{array}{c}
c \\
\varepsilon-1
\end{array}\right)}\right)\right)=0 \text {. }
$$

Obviously $s k^{i+1}\left(R_{c, \varepsilon}\right) \subseteq s k^{i+1}\left(\Delta_{\left(\begin{array}{c}c \\ \varepsilon-1\end{array}\right)}\right)$. We want to show $\tilde{H}_{i}\left(s k^{i+1}\left(R_{c, \varepsilon}\right)\right)=0$. Let $\beta$ be an $i$-cycle in $s k^{i+1}\left(R_{c, \varepsilon}\right)$. Since $\tilde{H}_{i}\left(s k^{i+1}\left(\Delta_{\left(\begin{array}{c}c \\ \varepsilon-1\end{array}\right)}\right)\right)=0$, there exists an 
$(i+1)$-chain $\eta$ in $s k^{i+1}\left(\Delta_{\left(\begin{array}{c}c \\ \varepsilon-1\end{array}\right)}\right)$ s.t. $\partial \eta=\beta$. Suppose $s p(\eta)=\bigcup_{j} F_{j}$, where $F_{j}$ are $(i+1)$-simplexes in $s k^{i+1}\left(\Delta_{\left(\begin{array}{c}c \\ \varepsilon-1\end{array}\right)}\right)$; consider now an $(i+1)$-chain $\psi$ in $s k^{i+1}\left(R_{c, \varepsilon}\right)$ whose support is $\bigcup_{j} \hat{F}_{j}$, where $\hat{F}_{j}=F_{j}$ if $F_{j} \subseteq s k^{i+1}\left(R_{c, \varepsilon}\right)$ and $\hat{F}_{j}$ is a cone on the border of $F_{j}$ if $F_{j} \nsubseteq \subseteq s k^{i+1}\left(R_{c, \varepsilon}\right)$, in such way that $\partial \psi=\beta$ (observe that in our cases such cones exist, in fact: $R_{c, \varepsilon}$ is the union of the (possibly degenerate) $(s-2)$ simplexes "obtained from the (possibly degenerate) $(s-1)$-simplexes of $\Delta_{\left(\begin{array}{c}c \\ \varepsilon-1\end{array}\right)}$ by taking off a vertex whose last coordinate is 0 "; in the case $i=0$ one can check that the 1-simplexes s.t. the last coordinate of a vertex and the last coordinate of the other vertex are 1, 1 or 1, 0 are contained in $R_{c, \varepsilon}$, while for a 1 -simplex $F$ s.t. the last coordinate of each vertex is 0 , there exists a cone, $\hat{F}$, on the border of $F$ with $\hat{F} \subseteq R_{c, \varepsilon}$, since $s \geq 3$; analogously case b)). Thus we proved $\tilde{H}_{i}\left(s k^{i+1}\left(R_{c, \varepsilon}\right)\right)=0$. Thus $\tilde{H}_{i}\left(R_{c, \varepsilon}\right)=0$.

Proof that $\mathcal{O}_{\mathbf{P}^{1} \times \ldots \times \mathbf{P}^{1}}(1, \ldots, 1)$ satisfies property $N_{2}$.

Lemma 18. Let $b \in \mathbf{N} A_{d}$, $\operatorname{deg} b=k, k \geq 4$ and $\varepsilon \in\left\{1, \ldots,\left[\frac{k}{2}\right]\right\}$. Every $1-$ cycle $\gamma$ in $\Delta_{\left(\begin{array}{l}b \\ \varepsilon\end{array}\right)}$ is homologous to a 1 -cycle in $F^{1}\left(\Delta_{b}\right)$ (which is $\subseteq \Delta_{\left(\begin{array}{l}b \\ \varepsilon\end{array}\right)}$ since $k-\varepsilon \geq 2$ ).

Proof. Obviously we can suppose $s p(\gamma) \subseteq s k^{1}\left(\Delta_{\left(\begin{array}{l}b \\ \varepsilon\end{array}\right)}\right)$. The proof is by induction on the cardinality of $\left(s p(\gamma) \cap s k^{0}\left(\Delta_{\left(\begin{array}{l}b \\ \varepsilon\end{array}\right)}\right)\right)-F^{1}\left(\Delta_{b}\right)$, i.e. we will prove that $\gamma$ is homologous to a 1-cycle $\tilde{\gamma}$ s.t.

$$
\sharp\left(\left(s p(\tilde{\gamma}) \cap s k^{0}\left(\Delta_{\left(\begin{array}{c}
b \\
\varepsilon
\end{array}\right)}\right)\right)-F^{1}\left(\Delta_{b}\right)\right)<\sharp\left(\left(s p(\gamma) \cap s k^{0}\left(\Delta_{\left(\begin{array}{c}
b \\
\varepsilon
\end{array}\right)}\right)\right)-F^{1}\left(\Delta_{b}\right)\right) .
$$

First we remark that if $P,\left(\begin{array}{l}a \\ 1\end{array}\right) \in s k^{0}\left(\Delta_{\left(\begin{array}{l}b \\ \varepsilon\end{array}\right)}\right)$ and $\left\langle P,\left(\begin{array}{l}a \\ 1\end{array}\right)\right\rangle \subseteq \Delta_{\left(\begin{array}{c}b \\ \varepsilon\end{array}\right)}$, then $P \in R_{b-a, \varepsilon}$ $(*)$.

In fact, $\left\langle P,\left(\begin{array}{l}a \\ 1\end{array}\right)\right\rangle \subseteq \Delta_{\left(\begin{array}{c}b \\ \varepsilon\end{array}\right)}$, then $P \in \Delta_{\left(\begin{array}{c}b-a \\ \varepsilon-1\end{array}\right)}$; we recall that $R_{b-a, \varepsilon}$ is

$$
\bigcup_{\substack{\alpha_{1}, \ldots, \alpha_{k-1} \in A_{d} \\
\text { s.t. } \alpha_{1}+\ldots+\alpha_{k-1}=b-a}} \bigcup_{\substack{i_{1}, \ldots, i_{k-2} \in\{1, \ldots, k-1\} \\
i_{l} \neq i_{m}}}\left\langle\left(\begin{array}{c}
\alpha_{i_{1}} \\
1
\end{array}\right), \ldots,\left(\begin{array}{c}
\alpha_{i_{\varepsilon-1}} \\
1
\end{array}\right),\left(\begin{array}{c}
\alpha_{i_{\varepsilon}} \\
0
\end{array}\right), \ldots,\left(\begin{array}{c}
\alpha_{i_{k-2}} \\
0
\end{array}\right)\right\rangle,
$$

i.e. $R_{b-a, \varepsilon}$ is the union of the (possibly degenerate) $(k-3)$-simplexes "obtained from the (possibly degenerate) $(k-2)$-simplexes of $\Delta_{\left(\begin{array}{c}b-a \\ \varepsilon-1\end{array}\right)}$ by taking off a vertex whose last coordinate is 0 "; then, if the last coordinate of $P$ is 1 , we may conclude at once that $P \in R_{b-a, \varepsilon}$; also if the last coordinate of $P$ is 0 , we may conclude that $P \in R_{b-a, \varepsilon}$, because the number of the vertices whose last coordinate is 0 in a (possibly degenerate) $(k-2)$-simplex of $\Delta_{\left(\begin{array}{c}b-a \\ \varepsilon-1\end{array}\right)}$ is $k-1-(\varepsilon-1) \geq 2$.

Now let $\left(\begin{array}{l}a \\ 1\end{array}\right) \in\left(s p(\gamma) \cap s k^{0}\left(\Delta_{\left(\begin{array}{l}b \\ \varepsilon\end{array}\right)}\right)\right)-F^{1}\left(\Delta_{b}\right),\left(a \in A_{d}\right)$. Intuitively we want to modify slightly $\gamma$ to obtain a homologous 1-cycle $\tilde{\gamma}$ passing through $\left(\begin{array}{l}a \\ 0\end{array}\right)$ instead of $\left(\begin{array}{l}a \\ 1\end{array}\right)$.

Let $\mathcal{S}_{\left(\begin{array}{c}a \\ 1\end{array}\right)}$ be the set of all 1 -simplexes $\tau$ of $\gamma$ s.t. $s p(\tau) \ni\left(\begin{array}{l}a \\ 1\end{array}\right)$. We can suppose that every 1-simplex $\tau \in \mathcal{S}_{\left(\begin{array}{c}a \\ 1\end{array}\right)}$ be s.t. $\operatorname{sp}(\tau)$ be a 1-simplex of $\Delta_{\left(\begin{array}{l}b \\ \varepsilon\end{array}\right)}$ and $\tau:[0,1] \rightarrow \Delta_{\left(\begin{array}{l}b \\ \varepsilon\end{array}\right)}$ be injective.

For $\tau \in \mathcal{S}_{\left(\begin{array}{c}a \\ 1\end{array}\right)}$, let $\tau^{\prime}:[0,1] \rightarrow \Delta_{\left(\begin{array}{l}b \\ \varepsilon\end{array}\right)}$ be a 1-simplex s.t. $\tau^{\prime}(\epsilon)=\tau(\epsilon)$ if $\tau(\epsilon) \neq\left(\begin{array}{l}a \\ 1\end{array}\right)$ and $\tau^{\prime}(\epsilon)=\left(\begin{array}{l}a \\ 0\end{array}\right)$ if $\tau(\epsilon)=\left(\begin{array}{l}a \\ 1\end{array}\right)$, for $\epsilon \in\{0,1\}$. Let $\alpha$ be the 1 cycle $\sum_{\tau \in \mathcal{S}\left(\begin{array}{c}a \\ 1\end{array}\right)}\left(-\tau+\tau^{\prime}\right)$. 
By remark $(*)$, we have that $s p(\alpha) \subseteq C$, where $C$ is the union of the two cones $\left\langle\left(\begin{array}{l}a \\ 1\end{array}\right), R_{b-a, \varepsilon}\right\rangle$ and $\left\langle\left(\begin{array}{l}a \\ 0\end{array}\right), R_{b-a, \varepsilon}\right\rangle$. Observe that $C \subseteq \Delta_{\left(\begin{array}{l}b \\ \varepsilon\end{array}\right)}$. We state that $H_{1}(C)=0$ : by Theorem 10, since $\mathcal{O}_{\mathbf{P}^{1} \times \ldots \times \mathbf{P}^{1}}(1, \ldots, 1)$ ( $d$ times) satisfies Property $N_{1} \forall d$, we have $\tilde{H}_{0}\left(\Delta_{g}\right)=0 \forall g \in \mathbf{N} A_{d}$ with $\operatorname{deg} g \geq 3, \forall d$ (this can easily be proved directly without using that $\mathcal{O}_{\mathbf{P}^{1} \times \ldots \times \mathbf{P}^{1}}(1, \ldots, 1)$ satisfies Property $\left.N_{1}\right)$; then $\tilde{H}_{0}\left(\Delta_{\left(\begin{array}{l}b-a \\ \varepsilon-1\end{array}\right)}\right)=0$; thus $\tilde{H}_{0}\left(R_{b-a, \varepsilon}\right)=0$ by Lemma 17; we have $\tilde{H}_{i}(C)=\tilde{H}_{i-1}\left(R_{b-a, \varepsilon}\right)$; thus $H_{1}(C)=$ $\tilde{H}_{0}\left(R_{b-a, \varepsilon}\right)=0$. Thus we have that $\alpha$ is homologous to 0 . Thus $\gamma$ is homologous to $\gamma+\alpha$.

Obviously the support of $\tilde{\gamma}:=\gamma+\alpha$ can be obtained from $s p(\gamma)$ by substituting $s p(\tau)$ with $s p\left(\tau^{\prime}\right) \forall \tau \in \mathcal{S}_{\left(\begin{array}{c}a \\ 1\end{array}\right)}$. Then

$$
\sharp\left(\left(s p(\tilde{\gamma}) \cap s k^{0}\left(\Delta_{\left(\begin{array}{c}
b \\
\varepsilon
\end{array}\right)}\right)\right)-F^{1}\left(\Delta_{b}\right)\right)<\sharp\left(\left(s p(\gamma) \cap s k^{0}\left(\Delta_{\left(\begin{array}{l}
b \\
\varepsilon
\end{array}\right)}\right)\right)-F^{1}\left(\Delta_{b}\right)\right) ;
$$

thus we conclude the proof of Lemma 18

In order to prove that $\mathcal{O}_{\mathbf{P}^{1} \times \ldots \times \mathbf{P}^{1}}(1, . ., 1)\left(d\right.$ times) satisfies $N_{2}$ for any $d$, we suppose (by induction) that $H_{1}\left(\Delta_{b}\right)=0 \forall b \in \mathbf{N} A_{d}$ with $\operatorname{deg} b=k, k \geq 4$, and we show that $H_{1}\left(\Delta_{\left(\begin{array}{c}b \\ \varepsilon\end{array}\right)}\right)=0$ for $\varepsilon \in\left\{1, \ldots,\left[\frac{k}{2}\right]\right\}$.

Cases $\varepsilon \leq k-3$. We know that every 1-cycle $\gamma$ in $\Delta_{\left(\begin{array}{l}b \\ \varepsilon\end{array}\right)}$ is homologous to a 1 -cycle in $F^{1}\left(\Delta_{b}\right)$ by Lemma 18. Thus, since $F^{2}\left(\Delta_{b}\right) \subseteq \Delta_{\left(\begin{array}{c}b \\ \varepsilon\end{array}\right)}$ and $H_{1}\left(F^{2}\left(\Delta_{b}\right)\right)=0$ (because, by induction hypothesis, $H_{1}\left(\Delta_{b}\right)=0$ ), we have that $H_{1}\left(\Delta_{\left(\begin{array}{l}b \\ \varepsilon\end{array}\right)}\right)=0$.

Cases $\varepsilon>k-3$. These cases are slightly more difficult. By Lemma 18 every 1cycle $\gamma$ in $\Delta_{\left(\begin{array}{c}b \\ \varepsilon\end{array}\right)}$ is homologous to a 1-cycle $\gamma^{\prime}$ in $F^{1}\left(\Delta_{b}\right)$. But in these cases we have not the inclusion $F^{2}\left(\Delta_{b}\right) \subseteq \Delta_{\left(\begin{array}{l}b \\ \varepsilon\end{array}\right)}$, thus we have to conclude the proof in another way.

Since $H_{1}\left(F^{2}\left(\Delta_{b}\right)\right)=0$, there exists a 2-chain $\mu$ in $F^{2}\left(\Delta_{b}\right)$ s.t. $\partial \mu=\gamma^{\prime}$. Let $s p(\mu)=\bigcup_{i} F_{i}, F_{i}$ triangles in $F^{2}\left(\Delta_{b}\right)$. Consider a 2-chain $\psi$ in $\Delta_{\left(\begin{array}{l}b \\ \varepsilon\end{array}\right)}$ whose support is $\bigcup_{i} \hat{F}_{i}$, where $\hat{F}_{i}$ is a cone $\subseteq \Delta_{\left(\begin{array}{c}b \\ \varepsilon\end{array}\right)}$ on the border of $F_{i}$ (there exists by Remark 15), in such way that $\partial \psi=\gamma^{\prime}$; thus $\left[\gamma^{\prime}\right]=0$ in $H_{1}\left(\Delta_{\left(\begin{array}{c}b \\ \varepsilon\end{array}\right)}\right.$, thus $[\gamma]=0$ in $H_{1}\left(\Delta_{\left(\begin{array}{l}b \\ \varepsilon\end{array}\right)}\right.$ ). Thus $H_{1}\left(\Delta_{\left(\begin{array}{l}b \\ \varepsilon\end{array}\right)}\right)=0$.

Proof that $\mathcal{O}_{\mathbf{P}^{1} \times \ldots \times \mathbf{P}^{1}}(1, \ldots, 1)$ satisfies property $N_{3}$.

Lemma 19. Let $b \in \mathbf{N} A_{d}$ with $\operatorname{deg} b=k$ and $\varepsilon \in\left\{1, \ldots,\left[\frac{k}{2}\right]\right\}$. If $k \geq 5$, every 2-cycle $\mu$ in $\Delta_{\left(\begin{array}{l}b \\ \varepsilon\end{array}\right)}$ is homologous to a 2-cycle in $F^{2}\left(\Delta_{b}\right)$ (which is $\subseteq \Delta_{\left(\begin{array}{c}b \\ \varepsilon\end{array}\right)}$ since $k-\varepsilon \geq 3)$.

Proof. Obviously we can suppose that $s p(\mu) \subseteq s k^{2}\left(\Delta_{\left(\begin{array}{c}b \\ \varepsilon\end{array}\right)}\right)$. The proof is by induction on the cardinality of $\left(s p(\mu) \cap s k^{0}\left(\Delta_{\left(\begin{array}{l}b \\ \varepsilon\end{array}\right)}\right)\right)-F^{2}\left(\Delta_{b}\right)$, i.e. we will prove that $\mu$ is homologous to a 2-cycle $\tilde{\mu}$ s.t. $\sharp\left(\left(s p(\tilde{\mu}) \cap s k^{0}\left(\Delta_{\left(\begin{array}{l}b \\ \varepsilon\end{array}\right)}\right)\right)-F^{2}\left(\Delta_{b}\right)\right)<$ $\sharp\left(\left(s p(\mu) \cap s k^{0}\left(\Delta_{\left(\begin{array}{l}b \\ \varepsilon\end{array}\right)}\right)\right)-F^{2}\left(\Delta_{b}\right)\right)$.

We remark that if $P, Q,\left(\begin{array}{l}a \\ 1\end{array}\right) \in \operatorname{sk}^{0}\left(\Delta_{\left(\begin{array}{l}b \\ \varepsilon\end{array}\right)}\right)$ and $\left\langle P, Q,\left(\begin{array}{l}a \\ 1\end{array}\right)\right\rangle \subseteq \Delta_{\left(\begin{array}{l}b \\ \varepsilon\end{array}\right)}$, then $\langle P, Q\rangle \subseteq$ $R_{b-a, \varepsilon}$.

In fact $\left\langle P, Q,\left(\begin{array}{l}a \\ 1\end{array}\right)\right\rangle \subseteq \Delta_{\left(\begin{array}{c}b \\ \varepsilon\end{array}\right)}$; then $\langle P, Q\rangle \subseteq \Delta_{\left(\begin{array}{c}b-a \\ \varepsilon-1\end{array}\right)}$. Since $R_{b-a, \varepsilon}$ is the union of the (possibly degenerate) $(k-3)$-simplexes "obtained from the (possibly degenerate) $(k-2)$-simplexes of $\Delta_{\left(\begin{array}{c}b-a \\ \varepsilon-1\end{array}\right)}$ by taking off a vertex whose last coordinate is $0 "$ and 
since the number of the vertices whose last coordinate is 0 in a (possibly degenerate) $(k-2)$-simplex of $\Delta_{\left(\begin{array}{c}b-a \\ \varepsilon-1\end{array}\right)}$ is $k-1-(\varepsilon-1) \geq 3$, we have $\langle P, Q\rangle \subseteq R_{b-a, \varepsilon}$.

Let $\mathcal{S}_{\left(\begin{array}{c}a \\ 1\end{array}\right)}$ be the set of all 2-simplexes $\tau:\langle(0,0),(0,1),(1,0)\rangle \rightarrow \Delta_{\left(\begin{array}{c}b \\ \varepsilon\end{array}\right)}$ of $\mu$ s.t. $\quad s p(\tau) \ni\left(\begin{array}{l}a \\ 1\end{array}\right)$. We can suppose that every 2-simplex $\tau \in \mathcal{S}_{\left(\begin{array}{c}a \\ 1\end{array}\right)}$ be s.t. $s p(\tau)$ be a 2-simplex of $\Delta_{\left(\begin{array}{l}b \\ \varepsilon\end{array}\right)}, \tau(\epsilon) \in s k^{0}\left(\Delta_{\left(\begin{array}{l}b \\ \varepsilon\end{array}\right)}\right)$ for $\epsilon \in\{(0,0),(0,1),(1,0)\}$ and $\tau$ : $\langle(0,0),(0,1),(1,0)\rangle \rightarrow \Delta_{\left(\begin{array}{l}b \\ \varepsilon\end{array}\right)}$ be injective.

For $\tau \in \mathcal{S}_{\left(\begin{array}{c}a \\ 1\end{array}\right)}$ let $\tau^{\prime}:\langle(0,0),(0,1),(1,0)\rangle \rightarrow \Delta_{\left(\begin{array}{c}b \\ \varepsilon\end{array}\right)}$ be a 2-simplex s.t. $\tau^{\prime}(\epsilon)=\tau(\epsilon)$ if $\tau(\epsilon) \neq\left(\begin{array}{l}a \\ 1\end{array}\right)$ and $\tau^{\prime}(\epsilon)=\left(\begin{array}{l}a \\ 0\end{array}\right)$ if $\tau(\epsilon)=\left(\begin{array}{l}a \\ 1\end{array}\right)$ for $\epsilon \in\{(0,0),(0,1),(1,0)\}$. Let $\alpha$ be the 2-cycle $\sum_{\tau \in \mathcal{S}_{\left(\begin{array}{c}a \\ 1\end{array}\right)}}\left(-\tau+\tau^{\prime}\right)$.

By remark (*), we have that $s p(\alpha) \subseteq C$, where $C$ is the union of the two cones $\left\langle\left(\begin{array}{l}a \\ 1\end{array}\right), R_{b-a, \varepsilon}\right\rangle$ and $\left\langle\left(\begin{array}{l}a \\ 0\end{array}\right), R_{b-a, \varepsilon}\right\rangle$. Observe that $C \subseteq \Delta_{\left(\begin{array}{l}b \\ \varepsilon\end{array}\right) \text {. We state that }}$ $H_{2}(C)=0$ : we have already proved that $\mathcal{O}_{\mathbf{P}^{1} \times \ldots \times \mathbf{P}^{1}}(1, \ldots, 1)$ satisfies Property $N_{2}$ i.e. $H_{1}\left(\Delta_{g}\right)=0 \forall g$ with $\operatorname{deg} g \geq 4$; thus $H_{1}\left(\Delta_{\left(\begin{array}{c}b-a \\ \varepsilon-1\end{array}\right)}\right)=0$; then $H_{1}\left(R_{b-a, \varepsilon}\right)=0$ by Lemma 17; we have $\tilde{H}_{i}(C)=\tilde{H}_{i-1}\left(R_{b-a, \varepsilon}\right)$; thus $H_{2}(C)=H_{1}\left(R_{b-a, \varepsilon}\right)=0$. Thus we have that $\alpha$ is homologous to 0 . Thus $\mu$ is homologous to $\mu+\alpha$.

The support of the cycle $\tilde{\mu}:=\mu+\alpha$ can be obtained from $\operatorname{sp}(\mu)$ by substituting $\operatorname{sp}(\tau)$ with $\operatorname{sp}\left(\tau^{\prime}\right) \forall \tau \in \mathcal{S}_{\left(\begin{array}{c}a \\ 1\end{array}\right)}$. Then

$$
\sharp\left(\left(s p(\tilde{\mu}) \cap s k^{0}\left(\Delta_{\left(\begin{array}{l}
b \\
\varepsilon
\end{array}\right)}\right)\right)-F^{2}\left(\Delta_{b}\right)\right)<\sharp\left(\left(s p(\mu) \cap s k^{0}\left(\Delta_{\left(\begin{array}{c}
b \\
\varepsilon
\end{array}\right)}\right)\right)-F^{2}\left(\Delta_{b}\right)\right) ;
$$

thus we conclude the proof of Lemma 19.

In order to prove that $\mathcal{O}_{\mathbf{P}^{1} \times \ldots \times \mathbf{P}^{1}}(1, \ldots, 1)$ ( $d$ times) satisfies $N_{3}$ for any $d$, we suppose (by induction) that $H_{2}\left(\Delta_{b}\right)=0 \forall b \in \mathbf{N} A_{d}$ with $\operatorname{deg} b=k, k \geq 5$ and we show that $H_{2}\left(\Delta_{\left(\begin{array}{l}b \\ \varepsilon\end{array}\right)}\right)=0$ for $\varepsilon \in\left\{1, \ldots,\left[\frac{k}{2}\right]\right\}$.

Cases $\varepsilon \leq k-4$. We have that every 2-cycle $\mu$ in $\Delta_{\left(\begin{array}{l}b \\ \varepsilon\end{array}\right)}$ is homologous to a 2cycle $\tilde{\mu}$ in $F^{2}\left(\Delta_{b}\right)$ by Lemma [19] Since $H_{2}\left(F^{3}\left(\Delta_{b}\right)\right)=0$ (because, by induction hypothesis, $\left.H_{2}\left(\Delta_{b}\right)=0\right)$, we have that $[\tilde{\mu}]=0$ in $H_{2}\left(F^{3}\left(\Delta_{b}\right)\right)=0$. Since in these cases $F^{3}\left(\Delta_{b}\right) \subseteq \Delta_{\left(\begin{array}{l}b \\ \varepsilon\end{array}\right)}$, we may conclude that $[\mu]=[\tilde{\mu}]=0$ in $H_{2}\left(\Delta_{\left(\begin{array}{l}b \\ \varepsilon\end{array}\right)}\right)$, thus $H_{2}\left(\Delta_{\left(\begin{array}{l}b \\ \varepsilon\end{array}\right)}\right)=0$.

Cases $\varepsilon>k-4$. We have that every 2-cycle $\mu$ in $\Delta_{\left(\begin{array}{l}b \\ \varepsilon\end{array}\right)}$ is homologous to a 2cycle $\tilde{\mu}$ in $F^{2}\left(\Delta_{b}\right)$ by Lemma 19. Since $H_{2}\left(F^{3}\left(\Delta_{b}\right)\right)=0$ (because $H_{2}\left(\Delta_{b}\right)=0$ ), we have that $[\tilde{\mu}]=0$ in $H_{2}\left(F^{3}\left(\Delta_{b}\right)\right)=0$. But in these cases we have not the inclusion $F^{3}\left(\Delta_{b}\right) \subseteq \Delta_{\left(\begin{array}{l}b \\ \varepsilon\end{array}\right)}$, thus we may not conclude at once. Since $H_{2}\left(F^{3}\left(\Delta_{b}\right)\right)=0$, there exists a 3-chain $\nu$ in $F^{3}\left(\Delta_{b}\right)$ s.t. $\partial \nu=\tilde{\mu}$. We have that $s p(\nu)=\bigcup_{i} F_{i}, F_{i}$ tetrahedrons in $F^{3}\left(\Delta_{b}\right)$. Consider a 3-chain $\psi$ in $\Delta_{\left(\begin{array}{l}b \\ \varepsilon\end{array}\right)}$ whose support is $\bigcup_{i} \hat{F}_{i}$, where $\hat{F}_{i}$ is a cone $\subseteq \Delta_{\left(\begin{array}{l}b \\ \varepsilon\end{array}\right)}$ on the border of $F_{i}$ (there exists by Remark [15), in such a way that $\partial \psi=\tilde{\mu}$; thus $[\tilde{\mu}]=0$ in $H_{2}\left(\Delta_{\left(\begin{array}{l}b \\ \varepsilon\end{array}\right)}\right)$, thus $[\mu]=0$ in $H_{2}\left(\Delta_{\left(\begin{array}{l}b \\ \varepsilon\end{array}\right)}\right)$. Then we may conclude that $H_{2}\left(\Delta_{\left(\begin{array}{l}b \\ \varepsilon\end{array}\right)}\right)=0$.

This completes the proof of Theorem 6 .

\section{Proof of Proposition 7}

Let $X$ and $Y$ be two projective varieties and $L$ a very ample line bundle on $X$ and $M$ a very ample line bundle on $Y$. Let $\left\{\sigma_{0}, \ldots, \sigma_{k}\right\}$ be a basis of $H^{0}(X, L)$ and 
let $\left\{s_{0}, \ldots, s_{l}\right\}$ be a basis of $H^{0}(Y, M)$; we can suppose $\exists \bar{y} \in Y$ s.t. $s_{0}(\bar{y}) \neq 0$, $s_{j}(\bar{y})=0$ for $j \neq 0$; let $t_{i, j}$ be the coordinates corresponding to $\left\{\sigma_{i} \otimes s_{j}\right\}_{i, j}$ of the embedding of $X \times Y$ by $\pi_{X}^{*} L \otimes \pi_{Y}^{*} M$ (where $\pi$. is the projection on $\cdot$ ) and let $t_{i}$ be the coordinates corresponding to $\left\{\sigma_{0}, \ldots, \sigma_{k}\right\}$ of the embedding of $X$ by $L$.

Remark 20. By setting $t_{i, j}=0$ for $j \neq 0$ in an equation of $X \times Y$ and then taking off the last index (a 0 ) of each variable, we get an equation of $X$ (to prove this, use $\bar{y})$.

Remark 21. Let $M$ be a graded module on $\mathbf{C}\left[x_{1}, \ldots, x_{n}\right]$ with a minimal set of generators of degree $s$; then a subset of elements of degree $s$ of $M$ can be extended to a minimal set of generators if and only if these elements are linearly independent on $\mathbf{C}$.

Proof of Proposition 7 . Suppose $L$ satisfies Property $N_{p-1}$ but not $N_{p}$. We want to show $\pi_{X}^{*} L \otimes \pi_{Y}^{*} M$ does not satisfy Property $N_{p}$; we can suppose $\pi_{X}^{*} L \otimes \pi_{Y}^{*} M$ satisfies Property $N_{p-1}$. Let $l_{m}$ and $q_{m}$ be the ranks of the $m$-module of a minimal free graded resolution respectively of $G(L)$ and of $G\left(\pi_{X}^{*} L \otimes \pi_{Y}^{*} M\right)$. Let $\left\{g_{j}^{m}\right\}_{j=1, \ldots, l_{m}}$ be a minimal set of generators of the $m$-module $E_{m}$ of a minimal resolution of $G(L)$,

$$
\ldots \rightarrow E_{m} \rightarrow E_{m-1} \rightarrow \ldots \rightarrow E_{0} \rightarrow G(L) \rightarrow 0 .
$$

Since $L$ satisfies Property $N_{p-1}$ but not $N_{p}$, there exists a syzygy $S$ of $\left(g_{1}^{p-1}, \ldots\right.$, $\left.g_{l_{p-1}}^{p-1}\right)$, s.t. $S$ is not generated by linear syzygies of $\left(g_{1}^{p-1}, \ldots, g_{l_{p-1}}^{p-1}\right)$. Add a 0 to the indices of the variables appearing in $S$ and call $\tilde{S}$ the so-obtained vector of polynomials; let $\tilde{S}^{\prime}=(\tilde{S}, 0, \ldots, 0)$ with 0 repeated $q_{p-1}-l_{p-1}$ times.

Obviously by adding a 0 to the indices of each variable appearing in the equations of $X$, we get equations of $X \times Y$ and by adding a 0 to the indices of every variable appearing in the syzygies of $X$ we get syzygies of $X \times Y$.

Add a 0 to the indices of the variables appearing in $g_{j}^{m}$ and call $\tilde{g}_{j}^{m}$ the soobtained vector of polynomials for $j=1, \ldots, l_{m}$; set $f_{j}^{1}=\tilde{g}_{j}^{1}$ for $j=1, \ldots, l_{1}$ and $f_{j}^{m}=\left(\tilde{g}_{j}^{m}, 0, \ldots, 0\right)\left(0\right.$ repeated $q_{m-1}-l_{m-1}$ times $)$ for $j=1, \ldots, l_{m}$ and $2 \leq m \leq$ $p-1 ; f_{j}^{m}$ for $j=1, \ldots, l_{m}$ are vectors of linear polynomials for $2 \leq m \leq p-1$ and they are quadratic if $m=1$. Thus, by induction on $m$ and by Remark 21, one can extend this set to a minimal set of generators $\left\{f_{j}^{m}\right\}_{j=1, \ldots, q_{m}}$, of the $m$-module of a minimal resolution of $G\left(\pi_{X}^{*} L \otimes \pi_{Y}^{*} M\right)$ for $m \leq p-1$ (we recall that we supposed $\pi_{X}^{*} L \otimes \pi_{Y}^{*} M$ satisfies Property $N_{p-1}$ ); we can do it in such way that, when we set $t_{i, j}=0$ for $j \neq 0$, we have that $f_{j}^{1}$ is zero for $j=l_{1}+1, \ldots, q_{1}$ and the $r$-th coordinate of $f_{j}^{m}$ is zero for $r \leq l_{m-1}$ and $j=l_{m}+1, \ldots, q_{m}$ (we can prove this by induction on $m$, by using Remark 20 for the case $m=1$ : it is sufficient to subtract linear combination of $f_{j}^{m}$ for $j=1, \ldots, l_{m}$ to $f_{j}^{m}$ for $\left.j=l_{m}+1, \ldots, q_{m}\right)$.

Obviously $\tilde{S}^{\prime}$ is a syzygy of $\left(f_{1}^{p-1}, \ldots, f_{q_{p-1}}^{p-1}\right)$.

If $\pi_{X}^{*} L \otimes \pi_{Y}^{*} M$ satisfies Property $N_{p}$, then $\tilde{S}^{\prime}$ would be generated by linear syzygies of $\left(f_{1}^{p-1}, \ldots, f_{q_{p-1}}^{p-1}\right)$.

We state that $\tilde{S}^{\prime}$ cannot be generated by linear syzygies of $\left(f_{1}^{p-1}, \ldots, f_{q_{p-1}}^{p-1}\right)$. In fact, if it were, say $\tilde{S}^{\prime}=\sum_{\alpha} S_{\alpha}\left(S_{\alpha}\right.$ linear syzygies of $\left.\left(f_{1}^{p-1}, \ldots, f_{q_{p-1}}^{p-1}\right)\right)$, we set $t_{i, j}=0$ for $j \neq 0$ in each member of the equality $\tilde{S}^{\prime}=\sum_{\alpha} S_{\alpha}$ and, by taking off the last index (a 0 ) of every variable and considering only the first $l_{p-1}$ coordinates of $S$ and $S_{\alpha}$, one would obtain that $S$ would be generated by linear syzygies of 
$\left(g_{1}^{p-1}, \ldots, g_{l_{p-1}}^{p-1}\right)$ (observe that by setting $t_{i, j}=0$ for $j \neq 0$ in $S_{\alpha}$ and taking the first $l_{p-1}$ coordinates, we get a syzygy of $\left.\left(f_{1}^{p-1}, \ldots, f_{l_{p-1}}^{p-1}\right)\right)$.

But $S$ cannot be generated by linear syzygies by assumption.

The line bundle $\mathcal{O}_{\mathbf{P}^{1} \times \mathbf{P}^{1} \times \mathbf{P}^{1}}(1,1,1)$ does not satisfy Property $N_{4}$; precisely the resolution, with the notation of Introduction, is

$$
0 \rightarrow S(-6) \rightarrow S(-4)^{9} \rightarrow S(-3)^{16} \rightarrow S(-2)^{9} \rightarrow S \rightarrow G \rightarrow 0 .
$$

This has been proved by Barcanescu and Manolache in $\mathrm{B}-\mathrm{M}$ and can be seen also by using the program Macaulay $\left[\mathrm{B}-\mathrm{S}\right.$ ] (to see only that $\mathcal{O}_{\mathbf{P}^{1} \times \mathbf{P}^{1} \times \mathbf{P}^{1}}(1,1,1)$ does not satisfy Property $N_{4}$, it is sufficient to use the autoduality of the resolution; see [B-M]).

From this and from Proposition 7 we deduce that $\mathcal{O}_{\mathbf{P}^{1} \times \ldots \times \mathbf{P}^{1}}(1, \ldots, 1)(d$ times) does not satisfy Property $N_{4}$ for $d \geq 3$. By also using Gallego-Purnapranja's Theorem 4 , we deduce that, if $a_{1}, \ldots, a_{d}$ are integer numbers with $a_{1} \leq a_{2} \leq \ldots \leq a_{d}$ and $a_{1}=\ldots=a_{k}=1$, the line bundle $\mathcal{O}_{\mathbf{P}^{1} \times \ldots \times \mathbf{P}^{1}}\left(a_{1}, \ldots, a_{d}\right)$ does not satisfy Property $N_{4}$ if $k \geq 3$ and it does not satisfy Property $N_{2 a_{k+1}+2 a_{k+2}-2}$ if $d-k \geq 2$.

With the same argument as in the Remark in part II, Section 2 of [Gr1-2] we deduce Corollary 8

\section{ACKNOWLEDGEMENTS}

I thank G. Ottaviani for several helpful conversations and above all for the valuable suggestion to study syzygies of Segre embeddings. I thank also G. Anzidei and C. Brandigi for some discussions and the referee for some comments.

\section{REFERENCES}

[B-M] S. Barcanescu, N. Manolache Betti numbers of Segre-Veronese singularities Rev. Roumaine Math. Pures Appl. 26 no.4, 549-565 (1981) MR 82j:13029

[B-S] D. Bayer, M. Stillman Macaulay: A system for computation in algebraic geometry and commutative algebra. It can be downloaded from math.columbia.edu/bayer/macaulay via anonymous ftp.

[C-M] A. Campillo, C. Marijuan Higher relations for a numerical semigroup Sem. Theorie Nombres Bordeaux 3, 249-260 (1991) MR 93d:13027

[C-P] A. Campillo, P. Pison L'ideal d'un semigroupe de type fini Comptes Rendus Acad. Sci. Paris Serie I, 316, 1303-1306 (1993) MR 94b:20055

[G-P] F.J. Gallego, B.P. Purnaprajna Some results on rational surfaces and Fano varieties J. Reine Angew Math. 538, 25-55 (2001)

[Gr1-2] M. Green Koszul cohomology and the geometry of projective varieties I,II J. Differ. Geom. 20, 125-171, 279-289 (1984) MR 85e:14022 MR 86j:14011

[Gr3] M. Green Koszul cohomology and geometry, in: M. Cornalba et al. (eds), Lectures on Riemann Surfaces, World Scientific Press (1989) MR 91k:14012

[G-L] M. Green, R. Lazarsfeld On the projective normality of complete linear series on an algebraic curve Invent. Math. 83, 73-90 (1986) MR 87g:14022

[J-P-W] T. Josefiak, P. Pragacz, J.Weyman Resolutions of determinantal varieties and tensor complexes associated with symmetric and antisymmetric matrices Asterisque 87-88, 109189 (1981) MR 83j:14044

[Las] A. Lascoux Syzygies des variétés determinantales Adv. in Math. 30, 202-237 (1978) MR 80j:14043

[O-P] G. Ottaviani, R. Paoletti Syzygies of Veronese embeddings Compositio Mathematica 125, 31-37 (2001) CMP 2001:09 
[P-W] P. Pragacz, J.Weyman Complexes associated with trace and evalutation. Another approach to Lascoux's resolution Adv. Math. 57, 163-207 (1985) MR 87f:14030

[St] B. Sturmfels, Gröbner bases and convex polytopes, University Lecture Series American Mathematical Society 8 (1996). MR 97b:13034

Dipartimento di Matematica "U. Dini", via Morgagni 67/A, 50134 Firenze, Italia

E-mail address: rubei@math.unifi.it 